\title{
ACCURACY OF CONE-BEAM COMPUTED TOMOGRAPHY (NEWTOM- GIANO) IN DETECTING ALVEOLAR BONE FENESTRATIONS WITH DIFFERENT VOXEL SIZES
}

\author{
Ahmed Mohamed Hossam*
}

\begin{abstract}
Objectives: The purpose of this study was to evaluate the accuracy and reliability of cone-beam computed tomography (CBCT) in the diagnosis of fenestrations defects with different voxel sizes.

Material and Methods: 128 Defects were created in eight dry skulls with a diameter of one, two, three and four millimeters on the buccal aspect, respectively in each quadrant. Measurements were obtained using a digital caliper. These were considered to be the gold standard. The skulls were scanned by CBCT (NewTom- Giano) at two settings: (a) Voxel size $0.3 \mathrm{~mm}$ Standard Resolution (SR) (b) voxel size $0.125 \mathrm{~mm}$ High Resolution (HR). The accuracy of the CBCT measurements was determined by comparing the mean of all radiographic measurements, using SAS software version 9.4 for data analysis with those of direct measurements and Duncan's Multiple Range Test.
\end{abstract}

Results: Statistical analysis for both SR and HR with the gold standard measurements for each defect on the skulls revealed statistically significant correlation between the radiographic measurements and real measurements $(\mathrm{P} \leq 0.05)$ indicating accuracy, also no statistically significant difference was found within the measurements accuracy of each group $(\mathrm{P}>0.05)$ with the different defects sizes. Duncan's multiple range test revealed that the HR voxel size $0.125 \mathrm{~mm}$ is of slightly higher accuracy.

Conclusion: Based on the results of the present study, CBCT (NewTom- Giano) has demonstrated high accuracy and reliability, in measuring different fenestration sizes with different resolution (HR $0.0125 \mathrm{~mm}$ and SR $0.300 \mathrm{~mm}$ voxels). Measurements were with same accuracy despite the slightly higher performance of HR $(0.125 \mathrm{~mm}$ voxel $)$

KEYWORDS: Cone-beam computed tomography, Fenestrations, Measurement Accuracy

\section{INTRODUCTION}

The success of periodontal therapy depends on many factors. An accurate image of the morphology of periodontal bone destruction is considered one of the most important factors for the differential therapeutic treatment plan. ${ }^{(1)}$ When the tooth roots are denuded of bone with the roots surface covered only by the periosteum and gingiva, this

*Lecturer of Radiology. Faculty of Dentistry October University of Modern Sciences and Art. Egypt 
is defined as fenestration. (2) Studies have shown that alveolar fenestration are common in different types of malocclusions with the possibility of gingival recession and additional bone loss. ${ }^{(3,4)}$ Until recently fenestrations were difficult to visualize by traditional 2-dimensional radiography due to the superimposition of contralateral cortical bony or dental structures. ${ }^{(5)}$

Cone beam computed tomography (CBCT) provides a lower dose and a lower cost alternative for many applications involving preoperative planning of dental implant as well as providing a three - dimensional (3D) image of the alveolar bone and identification of osseous defects. ${ }^{(6)}$

CBCT provides a sub-millimeter spatial resolution for the craniofacial complex images, with a scanning time which is comparable to that of a panoramic radiography. CBCT images are displayed in a matrix formed of individual blocks referred to as voxels (volume element). CBCT can produce images of maxillofacial structures with a wide range of voxel sizes. The voxel size may be as low as $0.125 \mathrm{~mm}$ in CBCT and smaller than that achieved with conventional CT units. A higher radiation dose is required to achieve a smaller voxel size to provide better image resolution. The voxel in CBCT is isotropic (uniform in all directions). The image quality is maintained by the isotropic voxels in CBCT in all three orthogonal planes (axial, sagittal, and coronal). ${ }^{(7,8,9)}$

CBCT software provides the tools that measure distances, angles, zoom, invert the gray scale and adjust contrast. Linear measurements are used often in presurgical implant planning for determining the exact amount of alveolar bone (height and width) and consequently size of the dental implants. The linear measurements could also be used in orthodontic analysis and definition of jaw tumor size. Studies showed that up to $94 \%$ of the CBCT measurements have been accurate, within $1 \mathrm{~mm} .^{(10)}$

Since CBCT can be used to evaluate alveolar bone morphology, some studies have been conducted to examine fenestrations with $\mathrm{CBCT}$, however, the accuracy of a CBCT scan for detecting alveolar bone fenestration was doubtful. ${ }^{(11)}$

The aim of this study was to evaluate the accuracy and reliability of CBCT (NewTom-Giano) in detecting alveolar bone defect (fenestration), using different CBCT resolutions $(0.300 \mathrm{~mm}$ and $0.125 \mathrm{~mm}$ voxel size) in comparison with direct measurements on dry human skulls.

\section{MATERIAL AND METHODS}

Eight dry human skulls for the study were provided, with no identification of age, sex, or race. Defects were created resembling fenestration four in each quadrant with a diameter of one, two, three and four millimeters on the buccal aspect of the maxillary and the mandibular arches. The defects were created of equal width and height. The created defects were measured by the means of a digital caliper of 0.01 accuracy and recorded as the gold standard measurement, which was saved to for later comparison with the measurements obtained from the CBCT scanning.

For obtaining the CBCT images each dry skull was placed with the median sagittal plane perpendicular to the horizontal plane, as recommended by the $\mathrm{CBCT}$ patient positioning protocol. Imaging was performed using the (NewTom-Giano) CBCT (Quantitative Radiology, Imola, Italy)figure 1.

Each skull was scanned twice with a $(11 \mathrm{x} 5 \mathrm{~cm})$ field of view (FOV):

1- Standard resolution (SR) scan: voxel size 0.300 $\mathrm{mm}$ (10.8 mAs, 90kVp) and a 3.6 second exposure time.

2- High resolution (HR) scan: voxel size $0.125 \mathrm{~mm}$ $(27 \mathrm{mAs}, 90 \mathrm{kVp})$ and a 9 second exposure time.

Obtained data were reconstructed using the CBCT software (Newtom-GIANO/VG3-Annex, version 7.2, Imola, Italy) for volumetric analysis. The images were displayed in all 3 orthogonal planes (axial, coronal, and sagittal). Cross-sectional images 
of the region of interest were generated with a pitch distance of $1 \mathrm{~mm}$ and a slice thickness of $1 \mathrm{~mm}$. Measurements from each skull of both resolutions were recorded and remeasured one-week later. The mean of the two readings were compared with the gold standard.

\section{Statistical analysis}

The SAS software version 9.4 was used for data analysis. Measurement accuracy was evaluated by comparing the mean of all radiographic measurements for each image with linear measurements. Duncan's Multiple Range Test was used to estimate the relationship between the real measurement using digital caliper and radiographic measurements using CBCT soft ware. A (P value of $\leq 0.05$ ) was used to assign statistical significance.

\section{RESULTS}

The mean and standard deviation of the radiographic measurements of both $\mathrm{HR}$ and SR from the gold standard are summarized in Table 1.

The mean of all the radiographic measurements was insignificantly larger than that of the real measurements, the mean of HR were $(1.125 \pm$ $0.116)$ for $1 \mathrm{~mm},(2.1 \pm 0.075)$ for $2 \mathrm{~mm},(3.1 \pm$ $0.075)$ for $3 \mathrm{~mm}$ and $(4.05 \pm 0.053)$ for $4 \mathrm{~mm}$, while that of SR were $(1.337 \pm 0.176)$ for $1 \mathrm{~mm},(2.287$ $\pm 0.064)$ for $2 \mathrm{~mm},(3.325 \pm 0.07)$ for $3 \mathrm{~mm}$ and $(4.350 \pm 0.169)$ for $4 \mathrm{~mm}$. Revealing a statistically significant correlation between the radiographic measurements and real measurements $(\mathrm{P} \leq 0.05)$ indicating accuracy.

Overall, there was no statistically significant differences $(\mathrm{P}>0.05)$ within the measurements accuracy of each group with the different defects sizes measured by the same voxel size as shown in table 2 .

Duncan's multiple range test revealed that the HR voxel size $0.125 \mathrm{~mm}$ is more accurate in measuring the different defect sizes than that of SR voxel size $0.300 \mathrm{~mm}$, despite its clinical insignificance as shown in table 3 .

TABLE (1) Mean and standard deviation of radiographic and real measurements

\begin{tabular}{|c|c|c|}
\hline gold standard & HR $(0.125 \mathrm{~mm})$ & SR $(0.300 \mathrm{~mm})$ \\
\hline size & Mean $\pm \mathrm{SD}(\mathrm{mm})$ & Mean $\pm \mathrm{SD}(\mathrm{mm})$ \\
\hline $1 \mathrm{~mm}$ & $1.125 \pm 0.116$ & $1.337 \pm 0.176$ \\
\hline $2 \mathrm{~mm}$ & $2.100 \pm 0.075$ & $2.287 \pm 0.064$ \\
\hline $3 \mathrm{~mm}$ & $3.100 \pm 0.075$ & $3.325 \pm 0.070$ \\
\hline $4 \mathrm{~mm}$ & $4.050 \pm 0.053$ & $4.350 \pm 0.169$ \\
\hline
\end{tabular}

TABLE (2) Mean difference and coefficient variance of HR and SR with different defects.

\begin{tabular}{|c|c|c|c|c|c|}
\hline \multicolumn{2}{|c|}{ HR $(0.125 \mathrm{~mm}) 1,2,3$ and $4 \mathrm{~mm}$} & \multicolumn{3}{c|}{ SR $(0.300 \mathrm{~mm}) 1,2,3$ and $4 \mathrm{~mm}$} \\
\hline Diff Mean & Coeff Var & Significance & Diff Mean & Coeff Var & Significance \\
\hline 0.0937 & 89.01578 & 0.3513 & 0.3250 & 40.3911 & 0.7976 \\
\hline
\end{tabular}

TABLE (3) Duncan's Multiple Range Test (Means with the same letter are not significantly different)

\begin{tabular}{|c|c|c|c|c|c|c|c|c|}
\hline & \multicolumn{2}{|c|}{$1 \mathrm{~mm}$} & \multicolumn{2}{c|}{$2 \mathrm{~mm}$} & \multicolumn{2}{c|}{$3 \mathrm{~mm}$} & \multicolumn{2}{c|}{$4 \mathrm{~mm}$} \\
\hline Resolution & * D G & $* * \mathrm{M}$ & $\mathrm{D} \mathrm{G}$ & $\mathrm{M}$ & $\mathrm{D} \mathrm{G}$ & $\mathrm{M}$ & $\mathrm{D} \mathrm{G}$ & $\mathrm{M}$ \\
\hline gold standard & A & 1 & A & 2 & A & 3 & A & 4 \\
\hline HR(0.125 mm) & A & 1.125 & A & 2.1 & A & 3.1 & A & 4.05 \\
\hline SR $(0.300 \mathrm{~mm})$ & B & 1.337 & B & 2.287 & B & 3.325 & B & 4.35 \\
\hline
\end{tabular}

*Duncan Grouping (D G), **Mean (M) 


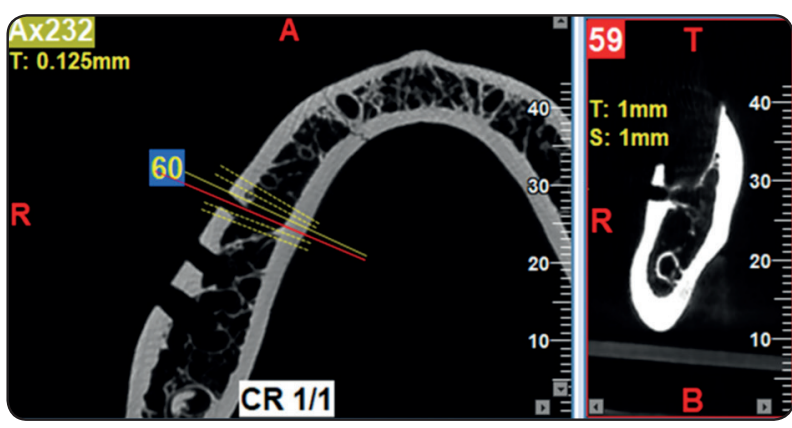

Fig (1) Fenestration defects at $0.125 \mathrm{~mm}$

\section{DISCUSSION}

Alveolar bone fenestration are common within patients with malocclusions, especially in the anterior region of skeletal Class III. ${ }^{(12)}$ Since the alveolar defect may complicate orthodontic treatment, awareness and precise diagnosis of such potential bone defect is necessary before treatment. ${ }^{(13)}$

In order to evaluate the accuracy and reliability of CBCT in detecting alveolar bone defect (fenestration), using different $\mathrm{CBCT}$ resolutions images $(0.125 \mathrm{~mm}$ and $0.300 \mathrm{~mm}$ voxel size) in comparison with direct measurements, the present study was conducted.

CBCT images were obtained using NewTomGiano and were measured using the software program (Newtom-GIANO/VG3-Annex, version 7.2, Imola, Italy) that was provided by the manufacturer.

Studies on the accuracy between the measurements obtained on the CBCT images and the direct measurements, requires the error should be $<1 \mathrm{~mm}$ to be considered accurate. ${ }^{(14)}$ Several studies have evaluated the accuracy of CBCT measurements and found varying results. In some, the measurements on CBCT images and direct measurements did not show statistically significant differences, whereas in others, despite the present differences, they were not considered clinically significant. ${ }^{(15,16)}$

The present study revealed a significant correlation $(\mathrm{P} \leq 0.05)$ between the measurement obtained from the CBCT images with different resolutions $(0.125 \mathrm{~mm}$ and $0.300 \mathrm{~mm}$ voxel size) and the direct measurements collected from the human dry skulls. These results are conferring with the studies done previously to assess the correlation between CBCT based measurements and actual measurements in human dry mandibles, human dry skulls or in patients. ${ }^{(17,18)}$

Also there were no significant difference $(\mathrm{P}>$ 0.05 ) found between the measurements obtained from images with a voxel size of $0.125 \mathrm{~mm}$ and $0.300 \mathrm{~mm}$. This was coherent with previous findings of the results of different voxel sizes effect on the accuracy of CBCT images. ${ }^{(19)}$ The mean of all the radiographic measurements was insignificantly larger than that of the real measurements, this was reported in previous studies as slight overestimation of the CBCT measurements compared to actual measurements. ${ }^{(20)}$ Duncan's Multiple Range Test revealed that the HR voxel size $0.125 \mathrm{~mm}$ is more accurate in measuring the different defect sizes than that of SR voxel size $0.300 \mathrm{~mm}$ despite its clinical insignificance.

\section{CONCLUSION}

Based on the results of the present study, CBCT (NewTom- Giano) has demonstrated high accuracy and reliability, in measuring different fenestration sizes with different resolution (HR $0.0125 \mathrm{~mm}$ and SR $0.300 \mathrm{~mm}$ voxels). Measurements were with same accuracy despite the slightly higher performance of HR (0.125 mm voxel) 


\section{REFERENCES}

1. X. Braun \& L. Ritter \& P.-M. Jervøe-Storm \& M. Frentzen. Diagnostic accuracy of CBCT for periodontal lesions. Clin Oral Invest 2014;18:1229-36.

2. Koke U, Sander C, Heinecke A, Muller HP. A possible influence of gingival dimensions on attachment loss and gingival recession following placement of artificial crowns. Int J Perio Rest Dent 2003;23:439-45.

3. Yagci A, Veli I, Uysal T, Ucar FI, Ozer T, Enhos S. Dehiscence and fenestration in skeletal Class I, II, and III malocclusions assessed with cone-beam computed tomography. Angle Orthod 2012;82: 67-74.

4. Sun LY, Wang B, Fang B. The prevalence of dehiscence and fenestration on anterior region of skeletal Class III malocclusions: a cone-beam CT study. Shanghai Kou Qiang Yi Xue 2013;22: 418-22.

5. Cynthia C. Leung, Leena Palomo, Richard Griffith and Mark G. Hansd. Accuracy and reliability of cone-beam computed tomography for measuring alveolar bone height and detecting bony dehiscences and fenestrations. Am J Orthod Dentofac Orthop 2010;137(4):109-19.

6. C. Vani, G. Raghavendra Prasad. Evaluation of validity of cone beam CT (CBCT) measurements compared to direct method on human dry mandibles. Int. Arc Integrated Med. (IAIM) 2017;4(10):59-66.

7. Fatemitabar SA, Nikgoo A. Multichannel computed tomography versus cone-beam computed tomography: Linear accuracy of in vitro measurements of the maxilla for implant placement. Int. J Oral Maxillofac Imp 2010;25:499-505.

8. Scarfe WC, Farman AG. What is cone-beam CT and how does it work? Dent Clin North Am 2008;52:707-30.

9. Araki K, Maki K, Seki K, Sakamaki K, Harata Y, Sakaino $\mathrm{R}$, et al. Characteristics of a newly developed dentomaxillofacial X-ray cone beam CT scanner (CB MercuRayTM): System configuration and physical properties. Dentomaxillofac Rad 2004;33:51-9.

10. Brown AA, Scarfe WC, Scheetz JP, Silveira AM, Farman AG. Linear accuracy of cone-beam CT derived 3D images. Angle Orthod 2009;79:150-7.
11. Liangyan Sun, Lina Zhang, Guofang Shen, Bo Wang, and Bing Fangc. Accuracy of cone-beam computed tomography in detecting alveolar bone dehiscences and fenestrations. Am J Ortho Dentofac Orthop 2015;147:313-23.

12. Kook YA, Kim G, Kim Y. Comparison of alveolar bone loss around incisors in normal occlusion samples and surgical skeletal class III patients. Angle Orthod 2012;82:645-52.

13. Rothe LE, Bollen AM, Little RM, Herring SW, Chaison JB, Chen CS, et al. Trabecular and cortical bone as risk factors for orthodontic relapse. Am J Orthod Dentofacial Orthop 2006;130:476-84.

14. Kamburo_glu K, Kilicx C, Ozen T, Yüksel SP. Measurements of mandibular canal region obtained by cone beam computed tomography: A cadaveric study. Oral Surg Oral Med Oral Path Oral Rad Endo 2009;107:e34-e42.

15. Moreira CR, Sales MA, Lopes PM, Cavalcanti MG. Assessment of linear and angular measurements on three-dimensional cone-beam computed tomographic images. Oral Surg Oral Med Oral Path Oral Rad Endo 2009;108:430-36.

16. Damstra J, Fourie Z, Huddleston Slater JJ, Ren Y. Accuracy of linear measurements from cone-beam computed tomography-derived surface models of different voxel sizes. Am J Orthod Dentofacial Orthop 2010;137:16.e1-16.e6.

17. Tarazona-Álvarez P, Romero-Millán J, Peñarrocha-Oltra D, Fuster-Torres MÁ, Tarazona B, Peñarrocha-Diago M. Comparative study of mandibular linear measurements obtained by cone beam computed tomography and digital calipers. J Clin Exp Dent 2014; 6: 271-4.

18. Stratemann SA, Huang JC, Maki K, Miller AJ, Hatcher DC. Comparison of cone beam computed tomography imaging with physical measures. Dentomaxillofac Rad 2014; 37: 80- 93 .

19. Mahkameh Moshfeghi, Mohammad Amin Tavakoli, EhsanTavakoli Hosseini, Ali Tavakoli Hosseini, Iman Tavakoli Hosseini. Analysis of linear measurement accuracy obtained by cone beam computed tomography (CBCT-NewTom VG). Dent Res J 2012;9(7):57-62.

20. Loubele M, Bogaerts R, Van Dijck E, Pauwels R, Vanheusden $\mathrm{S}$, Suetens $\mathrm{P}$, et al. Comparison between effective radiation dose of CBCT and MSCT scanners for dentomaxillofacial applications. Eur J Rad 2009; 71: 461-8. 